$>$ La chorée de Huntington est une maladie neurodégénérative héréditaire dominante caractérisée par l'apparition progressive de déficits moteurs, de troubles émotionnels et de l'humeur, ainsi que d'une détérioration des capacités intellectuelles. Elle aboutit irrémédiablement au décès des patients après environ 15-20 ans. Les études sur cette maladie ont pour la plupart avant tout cherché à identifier les mécanismes intrinsèques qui conduisent certaines populations de neurones à dysfonctionner, puis à dégénérer. Ce n'est que depuis les cinq dernières années que l'intérêt s'est aussi porté sur d'autres cellules du cerveau : les astrocytes. Cet article présente les arguments qui ont permis aujourd'hui d'admettre que le fonctionnement des astrocytes est également compromis dans la chorée de Huntington. Parmi les mécanismes possibles, la mutation responsable de cette maladie pourrait altérer la voie de signalisation du récepteur à l'EGF, qui régule en particulier la réponse des astrocytes à une atteinte des neurones. <

\title{
Les astrocytes dans la chorée de Huntington
}

Complices ou coupables de la mort neuronale?

Jean-Charles Liévens, Serge Birman

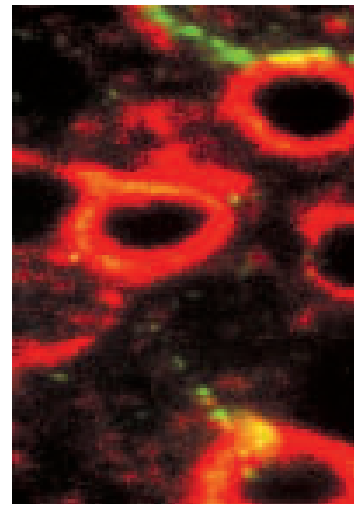

la protéine «Huntingtine ». Elle est due à un nombre trop élevé de triplets CAG (>36) dans le gène codant cette protéine [1]. Depuis l'identification du gène muté en 1993, la chorée de Huntington a fait l'objet d'études sur des thématiques aussi variées que l'apoptose, le trafic intracellulaire, l'excitotoxicité, la régulation de la transcription, la mitochondrie, les facteurs neurotrophiques ou le protéasome. Ces études avaient pour la plupart pour but direct de comprendre, à l'aide de modèles animaux, les mécanismes intrinsèques qui conduisent certaines populations neuronales à dysfonctionner ou dégénérer dans cette pathologie [2]. Plus récemment, des travaux ont abordé un aspect jusqu'alors négligé : le rôle des interactions cellulaires et des astrocytes.

\section{Le rôle des astrocytes}

Les cellules gliales représentent près de $90 \%$ des cellules du système nerveux et les astrocytes forment la principale population de cellules gliales dans le cerveau. Elles ont été qualifiées de cellules glues car on leur assignait autrefois un rôle passif consistant à combler les espaces vides entre les neurones. II aura fallu attendre cette 
dernière décennie pour que des études attribuent à ces cellules un rôle actif dans le développement et le fonctionnement normal du système nerveux $[3,4]$. Les astrocytes participent à la migration des neurones au cours du développement et à la formation de la barrière hématoencéphalique et hémato-liquide céphalorachidien. Ils secrètent des facteurs trophiques (bFGF, fibroblast growth factor; NGF, nerve growth factor...) qui conditionnent la survie et la plasticité des cellules nerveuses environnantes. Par ailleurs, les astrocytes ont une fonction métabolique essentielle: ils sont le lieu de stockage du glucose indispensable au fonctionnement des neurones et ils maintiennent I'homéostasie ionique du milieu extracellulaire via un ensemble de pompes ioniques. De plus, les astrocytes sont chargés d'éliminer certains neurotransmetteurs du milieu extracellulaire en les re-captant au travers de transporteurs transmembranaires spécifiques. Le rôle de

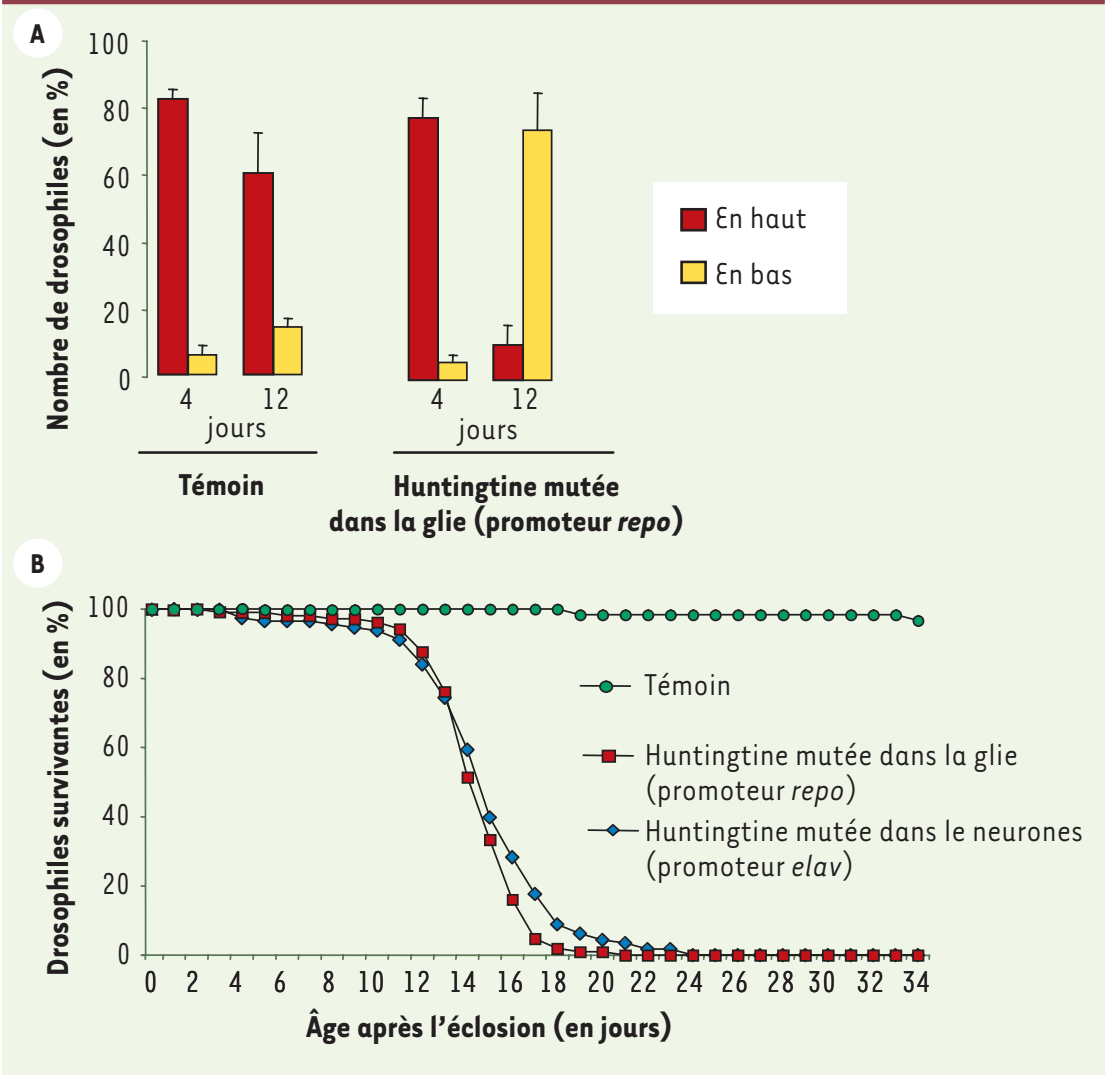

Figure 1. La présence de la protéine Huntingtine mutée dans les cellules gliales induit des déficits moteurs et réduit la durée de vie des drosophiles adultes. A. L'étude des déficits moteurs a été réalisée par test de géotaxie négative sur 35 drosophiles adultes (réparties en 3 groupes de 8/14 mouches) âgées de 4 ou 12 jours. Le nombre de drosophiles restant en bas (colonnes jaunes) ou atteignant le haut (colonnes rouges) d'un tube $(H: 18 \times D: 1,3 \mathrm{~cm})$ a été compté après une durée de 30 secondes. Les résultats représentent la moyenne des performances des trois groupes de drosophiles. B. L'analyse de survie a été réalisée sur 80-100 mouches adultes. L'expression de la protéine Huntingtine mutée a été spécifiquement dirigée dans la glie ou les neurones. Le promoteur repo permet d'affecter une large proportion de cellules gliales tandis que le promoteur elav induit une expression sélective dans les neurones. Les drosophiles exprimant la Huntingtine mutée dans les cellules gliales ou les neurones présentent une durée de vie également réduite comparée à celle des drosophiles témoins n'exprimant pas la protéine pathogène. ces transporteurs est multiple : limiter dans le temps et l'espace l'action des neurotransmetteurs, prévenir les processus de désensibilisation liés à la suractivation des récepteurs des neurotransmetteurs, recycler les neuromédiateurs et surtout éliminer certains neurotransmetteurs qui, en s'accumulant, pourraient s'avérer neurotoxiques. C'est en particulier le cas du glutamate, principal neurotransmetteur excitateur du cerveau, qui peut provoquer la mort des neurones lorsque son taux extracellulaire est anormalement élevé [5]. Certains astrocytes expriment en effet des transporteurs du glutamate de haute affinité afin de recapter le glutamate extracellulaire et de le convertir en glutamine, un acide aminé non toxique.

Deux dogmes, qui furent pendant longtemps attribués aux seuls neurones, ont récemment été élargis aux astrocytes. Bien qu'incapables de produire des potentiels d'action, les astrocytes sont aujourd'hui considérés comme des cellules «excitables » puisque des signaux internes ou externes peuvent modifier transitoirement leur taux calcique intracellulaire. De plus, les astrocytes expriment des récepteurs aux neurotransmetteurs et pourraient libérer des neuromédiateurs par exocytose ou par des canaux $[4,6]$. En particulier, les astrocytes possèdent des récepteurs au glutamate et peuvent, dans certaines conditions, libérer du glutamate par l'échangeur cystine/glutamate ou par exocytose de microvésicules semblables aux vésicules synaptiques. Ils régulent également l'activation des récepteurs au glutamate de type NMDA ( $N$-methyl-Daspartic acid) en libérant de la D-sérine dans le milieu extracellulaire. Enfin, lors de dommages cérébraux aigus (accident vasculaire cérébral, épilepsie...) ou progressifs (chorée de Huntington...), les cellules astrocytaires prolifèrent (astrogliose) et participent à la réparation des synapses, à la néo-neurogenèse et à la protection du tissu nerveux non affecté $[3,7]$.

\section{Les astrocytes dysfonctionnent dans la chorée de Huntington}

Comme les neurones, les cellules astrocytaires expriment le gène huntingtin 
[8-10]. Cependant, le rôle joué par ce gène dans le fonctionnement normal des astrocytes reste à élucider. En revanche, un faisceau de données récentes plaide en faveur d'anomalies de fonctionnement des astrocytes dans la chorée de Huntington. Chez les patients atteints par cette maladie, une réduction du nombre de sites de capture du glutamate a été décrite lors de l'analyse de prélèvements post-mortem de noyau caudé et de putamen (striatum), mais pas de cortex cérébral [11]. Par ailleurs, Arzberger et al. [12] ont montré que l'expression du principal transporteur astroglial du glutamate, $\varepsilon A A T 2$, est réduite dans le striatum en corrélation étroite avec la progression de la maladie. En fait, malgré une prolifération des astrocytes exprimant le transporteur (astrogliose), l'expression génique de EAAT2 pour chaque astrocyte

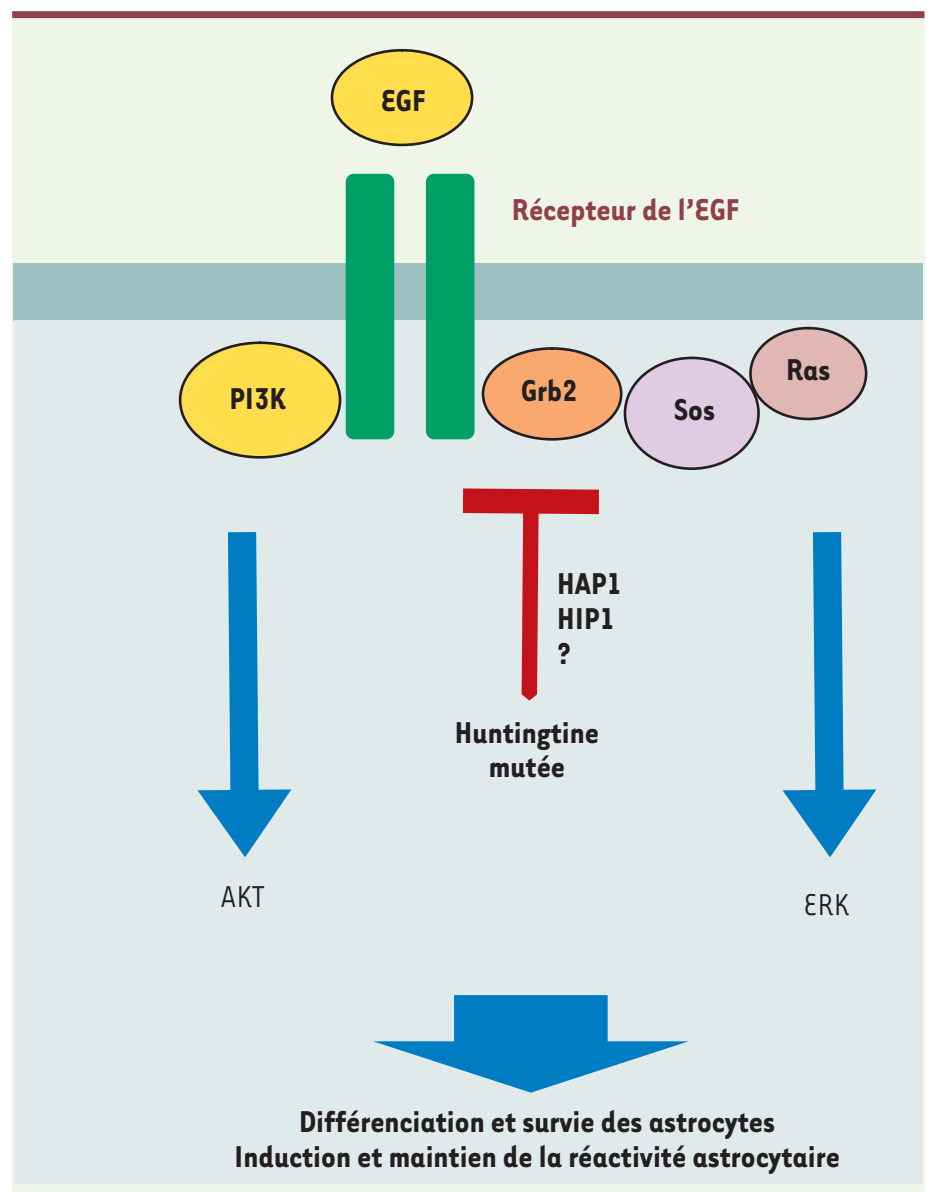

Figure 2. Représentation schématique de la voie de signalisation du récepteur de l'EGF. La fixation du ligand sur le récepteur à l'EGF conduit, entre autres, à l'activation de deux voies de signalisation intracellulaire : la voie phospho-inositide 3-kinase (PI3K) et la voie Ras au travers des protéines adaptatrices Grb2 et SOS. PI3K et Ras activent la protéine kinase B (AKT) ou la MAP kinase ERK, respectivement. L'activation du récepteur à l'EGF régule la différenciation et la survie des astrocytes et joue un rôle essentiel dans l'induction et le maintien de la réactivité astrocytaire. La présence de la protéine Huntingtine mutée altère la voie du récepteur à l'EGF en agissant soit directement sur des éléments de cette voie comme Grb2, soit indirectement au travers de protéines partenaires de la Huntingtine telles que HIPl et HAPl. est fortement altérée. Plus récemment, d'autres observations post-mortem ont montré que les astrocytes réactifs dans le striatum de patients présentent des taux anormalement élevés de la protéase apoptotique caspase-3 [13] et de la protéine de réparation de l'ADN poly(ADP-Ribose) polymérase ou PARP [14]. L'ensemble de ces résultats indique que les astrocytes sont affectés chez les patients atteints de chorée de Huntington. Deux études sur les modèles murins de la chorée de Huntington ont confirmé que l'expression de la protéine Huntingtine mutée altère l'expression de gènes spécifiques des astrocytes et, de ce fait, le transport du glutamate $[15,16]$, et ce en l'absence d'une dégénérescence substantielle des cellules gliales [9]. Une telle altération de la fonction des astrocytes pourrait soustendre transmission synaptique anormale observée chez ces souris [17] et contribuer à la mort des neurones par stimulation excessive des récepteurs du glutamate. La question se posait alors de savoir si cette perte de fonction était liée à la présence de la protéine Huntingtine mutée dans les astrocytes ou à des mécanismes plus indirects impliquant les neurones?

Pour tenter de répondre à cette question, un autre modèle animal de la chorée de Huntington, la drosophile, a été utilisé [2]. En effet, l'avancée des approches génétiques chez la drosophile permet d'exprimer une protéine sélectivement dans un type cellulaire choisi. C'est ainsi que nous avons exprimé la partie amino-terminale de la protéine Huntingtine mutée humaine dans presque toutes les cellules gliales avec le promoteur du gène repo. Une telle expression entraîne des déficits moteurs et une létalité importante des drosophiles adultes (Figure 1). On notera que les mouches meurent aussi vite lorsque la protéine pathogène est exprimée dans les cellules gliales avec repo ou dans les neurones avec le promoteur du gène elav. Sur le plan cellulaire, la présence de la protéine Huntingtine mutée dans les cellules gliales réduit progressivement l'expression de EAATl, l'unique transporteur de glutamate chez la drosophile [18], mais n'affecte pas significativement la survie de ces cellules [19].

Ces données apportent des arguments en faveur d'une action directe de la Huntingtine mutée sur le fonctionnement des astrocytes dans la chorée de Huntington. Les résultats du groupe de Xiao-Jiang Li [10] utilisant des cellules de mammifères en culture ont conforté cette hypothèse. Ces auteurs ont analysé in vitro l'expression du transporteur du glutamate EAAT2 dans des astrocytes exprimant la partie amino-terminale de la Huntingtine mutée. Dans cette situation, une sousexpression du transporteur est observée en l'absence d'une dégénérescence substantielle des astrocytes. 
De plus, l'étude montre que la présence de cellules gliales saines en co-culture retarde la dégénérescence de neurones exprimant la Huntingtine mutée. À l'inverse, l'expression de longues chaînes de résidus glutamine dans les cellules gliales favorise la mort des neurones sains, tandis que de courtes chaînes de résidus glutamine n'ont aucun effet. Enfin, une co-culture avec des astrocytes qui expriment la protéine Huntingtine mutée protège de manière moins efficace les neurones du cortex cérébral ou du striatum soumis à des processus excitotoxiques liés au glutamate. Des effets délétères sur les cellules gliales ont également été observés dans d'autres maladies liées à la présence de longues chaînes polyglutaminées dans d'autres protéines : c'est le cas dans l'ataxie spino-cérébelleuse de type 3 (SCA3) dans un modèle chez la drosophile [20] ou, plus récemment, l'ataxie spino-cérébelleuse de type 7 (SCA7) dans des souris transgéniques [21]. Dans ce dernier article, les auteurs ont exprimé la protéine Ataxine 7 mutée sélectivement dans la glie de Bergmann du cervelet et ont observé un déficit du transport du glutamate et une dégénérescence des neurones de Purkinje. L'ensemble de ces données suggère que l'expression de longues chaînes de résidus glutamine par les astrocytes altère leur capacité à éliminer le glutamate extracellulaire, contribuant ainsi à la pathogenèse de la chorée de Huntington ou d'autres maladies à polyglutamine. Mais il est fort probable que d'autres fonctions assurées par ces cellules soient également affectées dans ces situations pathologiques. Enfin, il n'est pas exclu que la diversité astrocytaire puisse jouer un rôle dans la spécificité des atteintes neuronales.

\section{Les mécanismes moléculaires du dysfonctionnement des astrocytes}

S’il semble clair que la protéine Huntingtine mutée peut altérer directement le fonctionnement des cellules gliales, il reste à élucider comment. Divers mécanismes ont été proposés pour expliquer le dysfonctionnement des neurones [2]. L'interaction de la protéine Huntingtine mutée avec des éléments du protéasome et des protéines chaperons inhiberait la dégradation des protéines mal conformées. La protéine Huntingtine mutée se lierait aussi à des protéines régulatrices de la transcription, modifiant ainsi l'expression de nombreux gènes. Enfin elle aurait des effets inhibiteurs sur les voies de signalisation contrôlant la croissance et la survie, et à l'inverse, des effets activateurs sur les voies de signalisation de l'apoptose. On peut envisager que les cellules gliales soient soumises aux mêmes mécanismes intrinsèques. Cependant, peu d'études ont été réalisées pour valider ou non cette hypothèse.

Notre intérêt s'est porté sur la voie de signalisation du récepteur du facteur de croissance épidermique (EGF), qui active en aval les voies Ras/MAP kinase (ERK) et phospho-inositide 3-kinase/AKT (Figure 2). En effet, les astrocytes expriment à leur surface ce récepteur, dont l'activation régule la différenciation ainsi que la survie des astrocytes dans les conditions normales, mais aussi joue un rôle critique dans l'induction et le maintien de la réactivité astrocytaire dans les conditions pathologiques [22]. Ainsi, le nombre de sites de recapture du glutamate serait régulé chez la drosophile et les mammifères par la voie de signalisation du récepteur à l'EGF [23]. Des données in vitro sur des cellules
PC12 en culture ont montré que la protéine Huntingtine mutée inhibe l'activation de ERK et AKT consécutive à la stimulation du récepteur à l'EGF [24, 25]. Nous avons donc fait l'hypothèse que la présence de longues chaînes de résidus glutamine modifierait l'expression de gènes essentiels pour le fonctionnement du système nerveux en altérant, entre autres, la voie de signalisation du récepteur à l'£GF dans les cellules gliales. En accord avec cette idée, l'expression de la protéine Huntingtine mutée in vivo dans les cellules gliales de drosophile prévient l'augmentation des taux de transporteur du glutamate induite par l'activation du récepteur à l'EGF, mais pas par l'activation de Ras ou ERK [19]. Ces données sont de plus étayées par des observations obtenues au niveau de l'œil composé de drosophile, dont le développement dépend étroitement de la voie de signalisation du récepteur à l'EGF. En effet, le phénotype induit par la suractivation du récepteur à l'EGF au niveau de l'œil de drosophile est significativement réduit lorsque de longues chaînes de résidus glutamine sont co-exprimées dans cette structure. En revanche, la malformation de l'œil qui résulte de l'expression de la forme active de ERK n'est pas affectée par la présence de longues chaînes de résidus glutamine [19]. L'ensemble de ces données suggère donc que la présence de la protéine Huntingtine mutée bloquerait la voie du récepteur à l'EGF en amont de la protéine Ras. Un des défis est à présent d'identifier les partenaires de cette voie de signalisation qui seraient la cible de la protéine Huntingtine mutée. La Huntingtine mutée pourrait agir directement sur des éléments de la voie du récepteur à l'EGF comme Grb2 [24], ou indirectement au travers de protéines partenaires de la Huntingtine comme HIPl (Huntingtin-interacting protein 1) et HAPl (Huntingtinassociated protein 1) $[25,26] . \diamond$

\section{SUMMARY}

Astrocytes in Huntington's chorea:

accomplice or guilty of the neuronal death?

Huntington's disease is an hereditary dominant neurodegenerative disorder clinically characterised by progressive motor deficits, personality changes, decreased mental capacity and death after about 15-20 years. Most studies are based on the research of intrinsic mechanisms that could be responsible for dysfunction and later degeneration of neuronal subsets. It is only in the last five years that more interest has been focused on another brain cell type : the astrocytes. This review presents evidence that astroglial function is also affected in Huntington's disease. Among the possible mechanisms, Huntington's disease mutation may alter the EGF receptor signaling pathway, that regulates the astrocytic response to neuronal injuries. $\diamond$ 


\section{REMERCIEMENTS}

Nous tenons à remercier la Fondation High $\rho$ pour son soutien à nos recherches sur les interactions neurones-glie dans la chorée de Huntington.

\section{RÉFÉRENCES}

1. The Huntington's Disease Collaborative Research Group. A novel gene containing a trinucleotide repeat that is expanded and unstable on Huntington's disease chromosomes. Cell $1993 ; 72: 971-83$.

2. Liévens JC, Birman S. La chorée de Huntington chez la drosophile et chez la souris: vers de nouvelles pistes thérapeutiques? Med Sci (Paris) $2003 ; 19: 593-9$.

3. Chneiweiss $\mathrm{H}$. Les astrocytes contrôlent la neurogenèse dans le système nerveux central. Med Sci (Paris) $2002 ; 18: 1065-6$.

4. Volterra A, Meldolesi J. Astrocytes, from brain glue to communication elements: the revolution continues. Nat Rev Neurosci 2005 ; 6 : 626-40.

5. Danbolt NC. Glutamate uptake. Prog Neurobiol $2001 ; 65$ : 1-105.

6. Matute C, Domercq M, Sanchez-Gomez MV. Glutamate-mediated glial injury: mechanisms and clinical importance. Glia $2006 ; 53: 212-24$.

7. Hansson $\varepsilon$, Ronnback L. Glial neuronal signaling in the central nervous system. FASEB J 2003 ; $17: 341-8$.

8. Singhrao SK, Thomas P, Wood JD, et al. Huntingtin protein colocalizes with lesions of neurodegenerative diseases: An investigation in Huntington's, Alzheimer's, and Pick's diseases. Exp Neurol 1998; $150: 213-22$.

9. Hebb MO, Denovan-Wright EM, Robertson HA. Expression of the Huntington's disease gene is regulated in astrocytes in the arcuate nucleus of the hypothalamus of postpartum rats. FASEB J $1999 ; 13: 1099-106$.

10. Shin JY, Fang ZH, Yu ZX, et al. Expression of mutant huntingtin in glial cells contributes to neuronal excitotoxicity. J Cell Biol $2005 ; 171$ : 1001-12.

11. Cross AJ, Slater P, Reynolds GP. Reduced high-affinity glutamate uptake sites in the brains of patients with Huntington's disease. Neurosci Lett 1986; 67 : 198-202.

12. Arzberger T, Krampfl K, Leimgruber S, Weindl A. Changes of NMDA receptor subunit (NRI, NR2B) and glutamate transporter (GLT1) mRNA expression in Huntington's disease-an in situ hybridization study. J Neuropathol Exp Neurol 1997 ; 56 : 440-54.

13. Hermel $\varepsilon$, Gafni J, Propp SS, et al. Specific caspase interactions and amplification are involved in selective neuronal vulnerability in Huntington's disease. Cell Death Differ 2004 ; $11: 424-38$.

14. Vis JC, Schipper $\varepsilon$, de Boer-van Huizen RT, et al. Expression pattern of apoptosis-related markers in Huntington's disease. Acta Neuropathol (Berl) 2005 ; 109 : 321-8.
15. Lievens JC, Woodman B, Mahal A, et al. Impaired glutamate uptake in the R6 Huntington's disease transgenic mice. Neurobiol Dis $2001 ; 8: 807-21$.

16. Behrens PF, Franz P, Woodman B, et al. Impaired glutamate transport and glutamate-glutamine cycling: downstream effects of the Huntington mutation. Brain 2002; 125 : 1908-22.

17. Nicniocaill B, Haraldsson B, Hansson 0 , et al. Altered striatal amino acid neurotransmitter release monitored using microdialysis in R6/1 Huntington transgenic mice. Eur J Neurosci 2001 ; 13 : 206-10.

18. Rival T, Soustelle L, Strambi C, et al. Decreasing glutamate buffering capacity triggers oxidative stress and neuropil degeneration in the Drosophila brain. Curr Biol 2004 ; 14 : 599-605.

19. Liévens JC, Rival T, Iche M, Chneiweiss H, Birman S. Expanded polyglutamine peptides disrupt $\varepsilon G F$ receptor signaling and glutamate transporter expression in Drosophila. Hum Mol Genet 2005 ; 14 : 713-24.

20. Kretzschmar D, Tschape J, Bettencourt Da Cruz A, et al. Glial and neuronal expression of polyglutamine proteins induce behavioral changes and aggregate formation in Drosophila. Glia $2005 ; 49: 59-72$.

21. Custer SK, Garden GA, Gill N, et al. Bergmann glia expression of polyglutamine-expanded ataxin-7 produces neurodegeneration by impairing glutamate transport. Nat Neurosci 2006 ; 9 : 1302-11.

22. Junier MP. What role(s) for TGFalpha in the central nervous system? Prog Neurobiol $2000 ; 62: 443-73$

23. Figiel M, Maucher T, Rozyczka J, et al. Regulation of glial glutamate transporter expression by growth factors. Exp Neurol 2003 ; 183: 124-35.

24. Song C, Perides G, Liu YF. Expression of full-length polyglutamineexpanded Huntingtin disrupts growth factor receptor signaling in rat pheochromocytoma (PC12) cells. J Biol Chem 2002 ; 277 : 6703-7.

25. Li SH, Yu ZX, Li CL, et al. Lack of huntingtin-associated protein-1 causes neuronal death resembling hypothalamic degeneration in Huntington's disease.J Neurosci 2003 ; $23: 6956-64$.

26. Bradley SV, Holland EC, Liu GY, et al. Huntingtin interacting protein 1 is a novel brain tumor marker that associates with epidermal growth factor receptor. Cancer Res $2007 ; 67: 3609-15$.

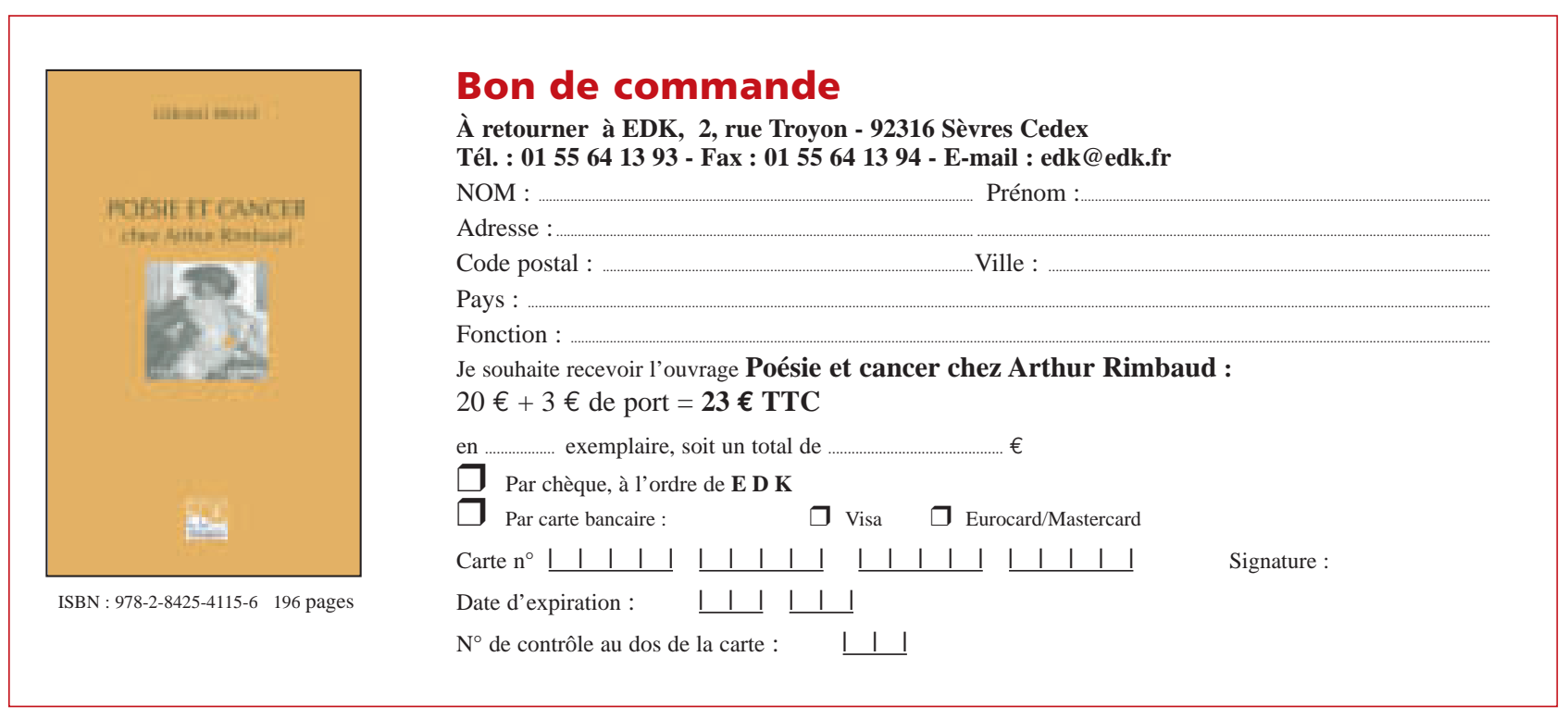

TIRÉS À PART

S. Birman 